\title{
Tratamiento temprano de malo- clusión II division 2: Reporte de un caso
}

Silva-Esteves Raffo JF, Amez-Atapoma J, Bustinza-Gómez P. Tratamiento temprano de maloclusión II division 2: Reporte de un caso. Rev Estomatol Herediana. 2008; 18(2):118-122.

RESUMEN

Se puede iniciar el tratamiento temprano de la maloclusión clase II división 2 en la dentición mixta, usando diferentes tipos de aparatología, el objetivo en esa fase es lograr una función muscular equilibrada, una relación molar clase I y mejorar el entrecruzamiento vertical y horizontal. Se reporta el caso de un niño de 9 años 4 meses, en dentición mixta primera fase con maloclusión clase II división 2 y discrepancia alveolo dentaria superior de $-7,3 \mathrm{~mm}$ e inferior de $-8,4 \mathrm{~mm}$. El tratamiento incluyó el uso de una placa de expansión con plano de mordida anterior, aparato extraoral ortodóntico de tracción cervical y lip bumper, obteniendo resultados satisfactorios en 18 meses.

Palabras clave: MALOCLUSIÓN DE ANGLE CLASE II / TÉCNICA DE EXPANSIÓN PALATINA / APARATOS DE TRACCIÓN EXTRAORAL.

Early treatment of malocclusion class II division 2: a case report

ABSTRACT

The early treatment of class II div 2 malocclusion can be started in the mixed dentition stage using different types of appliances. The objective in that phase is to obtain a class I molar relation, to improve the overjet and overbite, and to obtain a good perioral muscular function. It is reported the case of a 9 year 4 month boy, in first phase of mixed dentition, with class II division 2 malocclusion and space discrepancy of $-7.3 \mathrm{~mm}$ in maxilla and $-8.4 \mathrm{~mm}$ in mandible. The treatment included the use of an expansion plate with an anterior bite plane, a cervical headgear and a lip bumper, obtaining satisfactory results in 18 months.

Key words: ANGLE CLASS II MALOCCLUSION / PALATAL EXPANSION TECHNIQUE EXTRAORAL TRACTION APPLIANCES.

\section{Introducción}

Una de las estrategias para el tratamiento de la maloclusión clase II preconiza la intervención durante los años de preadolescencia (8-11 años) con objetivos limitados que incluyen corrección de la distoclusión de la molar, mejorar el entrecruzamiento vertical y horizontal y el alineamiento de los incisivos. La primera fase llamada "tratamiento temprano" es seguida por una segunda fase definitiva durante la adolescencia, para finalizar y detallar la oclusión (1). Esta forma de tratamiento ortodóntico parece ser más efectiva que tratarla más tarde, debido a que los tejidos del complejo craneofacial pueden ser más adaptables en una edad más joven y también debido al mayor grado de colaboración de los pacientes preadolescentes comparados con los adolescentes $(1,2)$.

La maloclusión clase II división 2, fue definida por primera vez por Angle en 1899 como una maloclusión en que los molares y caninos están en distoclusión y los incisivos centrales superiores se encuentran retroinclinados. Tiene una incidencia de 1,5 a 7\%, y su etiología generalmente genética o debida a factores como línea labial alta, hiperactividad labial y fuerzas masticatorias aumentadas $(3,4)$.

La maloclusión clase II división 2 se caracteriza por tener un biotipo mesofacial o braquifacial. Los pacientes presentan una cara más armónica que la división 1 , muchos muestran una convexidad facial normal y perfiles agradables pudiendo ser rectos o ligeramente convexos, sus ramas mandibulares son normales o largas y tienen buen potencial de crecimiento mandibular, siendo a veces muy semejante al paciente con maloclusiones clase I. La posición e inclinación de los incisivos es tal que los centrales superiores se encuentran inclinados hacia palatino, aunque a veces son los cuatro los que se encuentrán de esta for-
José F. Silva-Esteves Raffo' Jacquelyn Amez Atapoma ${ }^{2}$ Paola G. Bustinza Gómez ${ }^{2}$

Docente del Departamento Académico de Estomatología del Niño y del Adolescente. ${ }^{2}$ Residente del Programa de Especialización en Odontología Pediátrica.

Facultad de Estomatología. Universidad Peruana Cayetano Heredia.

\section{Correspondencia}

Fernando Silva Esteves Raffo

Calle San Martin 351 Of. 601 - Lima 18, Perú Teléfono: $446-7857$

e-mail: fernandoser@speedy.com

Recibido : 10 de noviembre del 2008

Aceptado : 15 de diciembre del 2008

ma. El overjet es normal o levemente aumentado y con frecuencia se observan mordidas profundas cuando no hay contacto interincisal debido a las condiciones fisiológicas y biotipo facial (5-7).

\section{Reporte de Caso}

Paciente masculino de 9 años 4 meses de edad, con diagnóstico de asma controlada y alergia al polvo, que acude al servicio de Odontología Pediátrica de la Clínica Estomatológica Central de la Universidad Peruana Cayetano Heredia, con motivo de consulta "veo los dientes de mi hijo medio salidos, muerde mal".

El examen clínico extraoral muestra un paciente mesocéfalo, mesofacial, perfil óseo convexo, normodivergente, con simetría facial conservada e hipertonicidad del labio inferior (Fig. 1).

Al examen clínico intraoral se observa a ambos maxilares de forma cuadrangular apiñados con rela- 


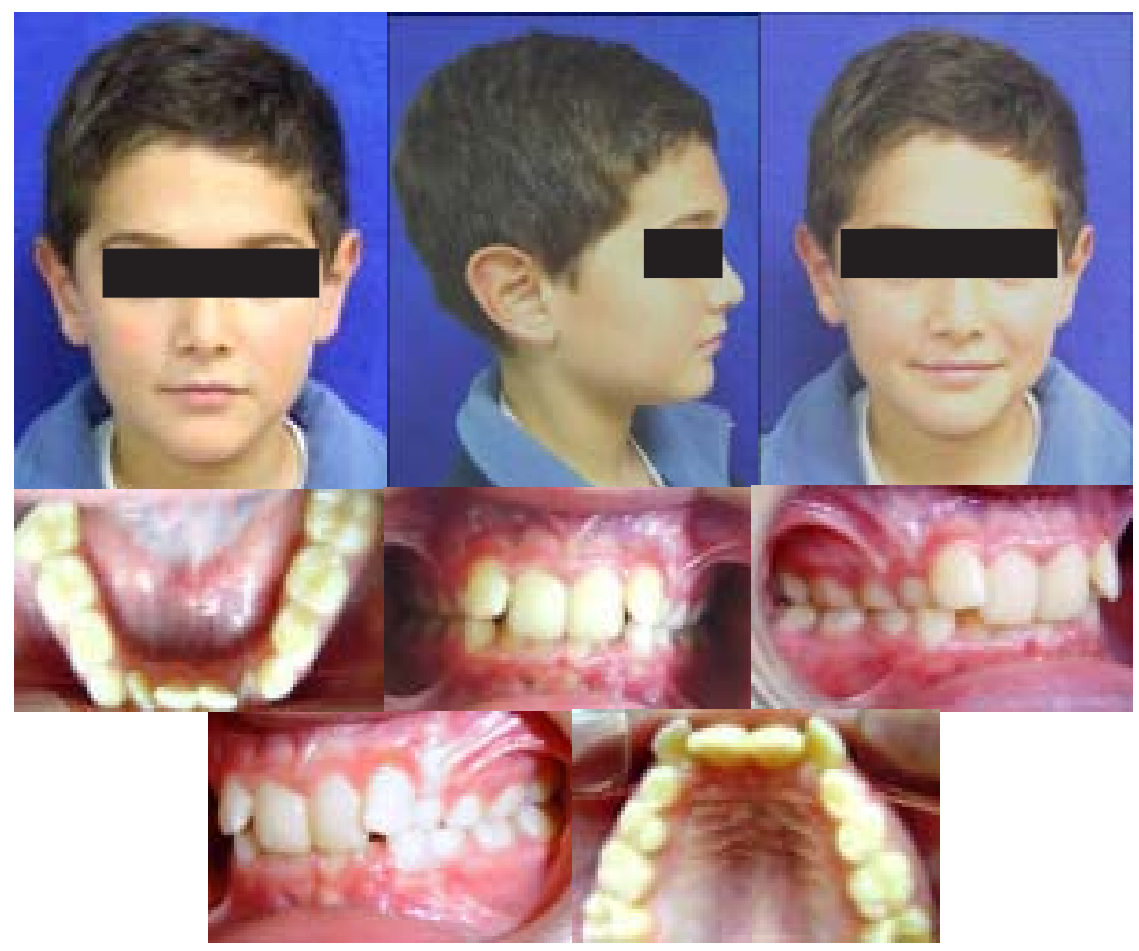

Fig. 1. Fotos iniciales.

ción molar derecha clase II e izquierda clase I, relación canina derecha clase II e izquierda clase I, entrecruzamiento vertical de $85 \%$ y horizontal de $1 \mathrm{~mm}$. Se aprecia mordida profunda anterior, apiñamiento dentario anterosuperior y anteroinferior, giroversión de la pza 42 e inclinaciones de piezas $11(\mathrm{P}) ; 21(\mathrm{P})$; 12(V); 22(V) (Fig. 1).

El análisis cefalométrico muestra relación esquelética clase I, hipodivergente y en normorotación; plano oclusal inclinado, incisivo superior palatinizado y retruido e incisivo inferior palatinizado con respecto a planos de referencia.

En el análisis de modelos de estudio las distancias iniciales bicanina superior es de $25,5 \mathrm{~mm}$ y la inferior de $24,5 \mathrm{~mm}$ y bimolar entre $16 / 26$ de $45 \mathrm{~mm}$ y $36 / 46$ de $41 \mathrm{~mm}$. Al análisis de Moyers se registró una discrepancia alveolodentaria (DAD) superior e inferior negativa severa de $-7,3 \mathrm{~mm}$ y $-8,4 \mathrm{~mm}$ respectivamente.

Estableciéndose como diagnós-

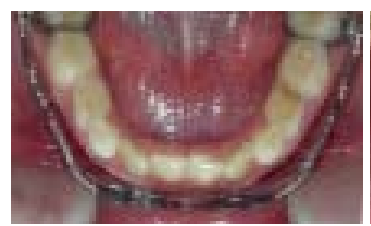

Fig. 2. Fotos con tornillo de expansión y lip bumper.

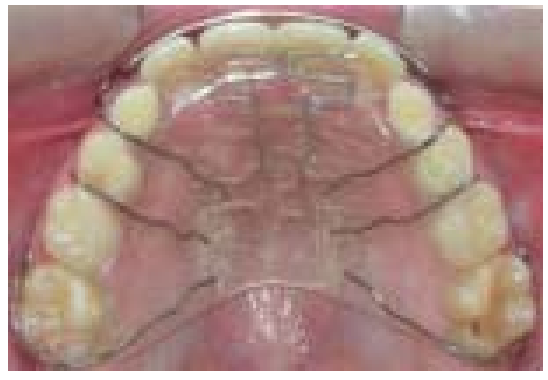

Fig. 3. Foto con placa de contensión.

hipertónico.

\section{Tratamiento}

El objetivo del tratamiento temprano de la maloclusión clase II división 2 fue corregir la distoclusión de la molar, alinear los incisivos superiores y mejorar el entrecruzamiento vertical y horizontal. El tratamiento consistió en el uso de una placa de Hawley superior activa con tornillo de expansión simétrico, resortes para las piezas 11 y 21 y plano de levantamiento de mordida anterior. Para el arco inferior se utilizó lip bumper con bandas en las piezas 36 y 46 . Una vez lograda la expansión requerida se procedió a la instalación de un arco extraoral (AEO) ortodóntico de tracción cervical.

La placa de expansión fue activada $2 / 4$ de vuelta por semana para una expansión palatina lenta. Al cabo de cinco meses de la instalación se observó que el tornillo de expansión había logrado la máxima apertura por lo que se bloqueó con acrílico de curado rápido y al mes se procedió a iniciar la activación de los resortes para vestibularizar las piezas 11 y 21 (Fig. 2). Después de dos meses se colocó una placa Hawley como aparato de contención (Fig. 3).
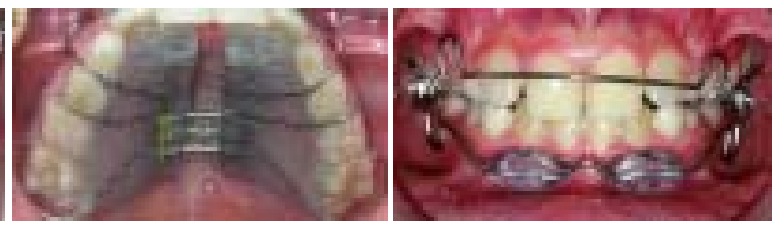

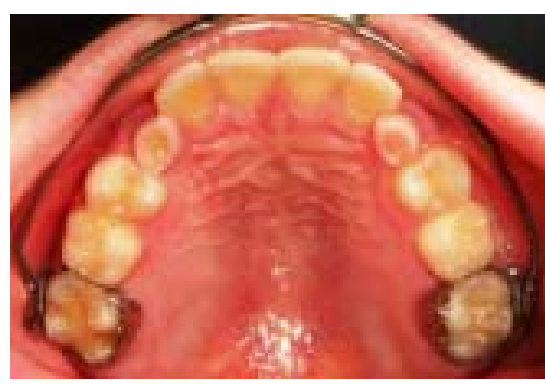

Fig. 4. Foto con arco extraoral. 
Luego de un año y dos meses de tratamiento, se decide retirar la aparatología instalada y se procede a la instalación del AEO cervical para distalizar las primeras molares permanentes superiores, crear espacio para aliviar discrepancia alveolo-dentaria superior, mejorar relación molar y guiar la erupción dentaria sucedánea (Fig. 4).

A los cinco meses de instalado el AEO y un año siete meses de tratamiento, se observa una relación molar clase I, alineamiento de incisivos y OB y OJ controlados (Fig. $5)$.

\section{Discusión}

La maloclusión II división 2 descrita por Angle es poco frecuente, siendo su prevalencia en Latinoamérica de un 0 a 5\% (8). Dentro de las características principales desde el punto de vista de la cefalometría, no sólo tiene un patrón patognomónico dental sino atributos esqueletales, sagitales y especialmente verticales que la diferencian de la clase I y clase II división I. Entre estas características destacan: la longitud mandibular corta y su posición sagital retruida, mentón prominente, las relaciones mandibulares antero posterior son similares a la clase I, la altura facial posterior está alargada, el vector de crecimiento mandibular está horizontalmente orientado, plano mandibular plano, patrón facial hipodivergente, ángulo gonial agudo, la longitud de la base craneal anterior es normal, los incisivos centrales superiores están en una pronunciada retroinclinación. Los incisivos inferiores tienen una relativa inclinación normal con relación al plano mandibular pero están retroinclinados a varios planos faciales. El ángulo interincisal es obtuso y el overbite es profundo debido a la rotación mandibular horaria $(2,9)$.

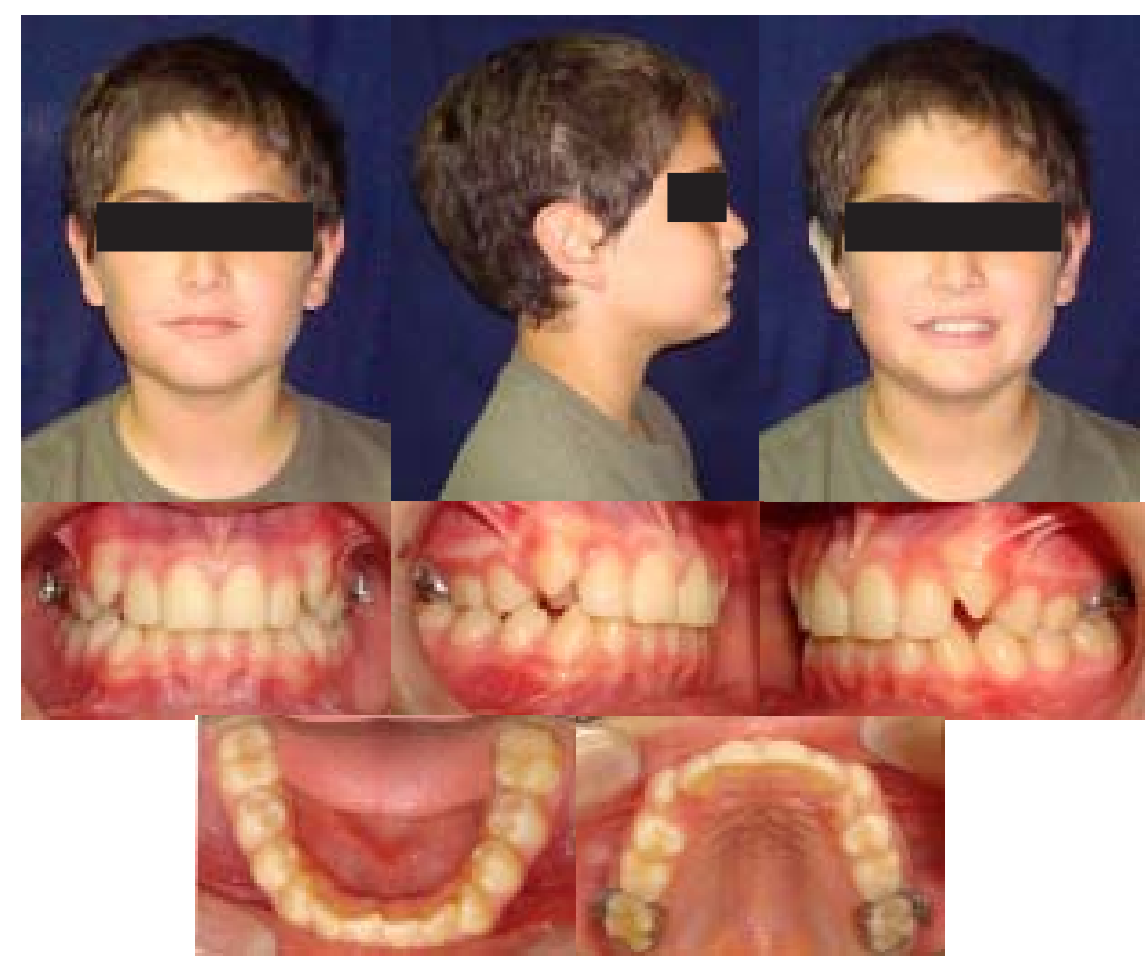

Fig. 5. Fotos finales.

Aunque no exista una regla de oro para el diagnóstico diferencial, la relación sagital de la mandíbula tiene un impacto en la elección del tratamiento. Los tratamientos recomendados incluyen la distalización de molares por medio de diferentes tipos de arcos de tracción, elásticos clase II, sistemas de aparatología fija como $2 \times 4$, arcos utilitarios, Twin blocks, planos de mordida con tornillos de expansión, lip bumper combinados con placas de mordida, para mencionar algunos $(8,10,11)$.

En el estudio realizado en el 2007 por Korn y Melsen (12), en 40 pacientes con dentición mixta y maloclusión clase II, usaron un aparato lip bumper combinado con una placa de mordida, en donde el lip bumper causó una distalización de las molares, incrementó el perímetro de arco y alineó los incisivos. La combinación del lip bumper con la placa de mordida tuvieron una influencia favorable en el desarrollo oclusal en niños con relaciones molares clase II y discrepancia de espacio.

En este caso reportado usamos durante un año el lip bumper, para corregir el problema funcional del labio inferior y la discrepancia alveolodentaria inferior, mejorando el apiñamiento dentario. Esto ocurrió por la expansión lograda con este dispositivo, la distancia bicanina inferior aumento de $24,5 \mathrm{~mm}$ a $26 \mathrm{~mm}$ y la distancia bimolar de $41 \mathrm{~mm}$ a $43 \mathrm{~mm}$. Asimismo, la longitud de arco se incremento de $29 \mathrm{~mm}$ a $33 \mathrm{~mm}$ luego del tratamiento, siendo este un aumento significativo. También se logró una ligera distoinclinación de la primera molar inferior permanente.

Murphy et al. (2002) (13), en un estudio hecho en 44 pacientes adolescentes con problemas de apiñamiento inferior, lograron una expansión del arco, siendo mayor durante el inicio del tratamiento, el 50\% de esta expansión ocurrió durante los primeros 100 días de tratamiento y el $90 \%$ de la expansión total dentro de los primeros 300 días. 
El uso de aparatos funcionales para corregir maloclusiones II división 2 en el tratamiento temprano es efectivo, evitándose la recidiva, así lo confirma un estudio hecho por Ferrazinni (2008) (14), quien evaluó la efectividad y estabilidad a largo plazo del tratamiento temprano con aparatos removibles en 20 años en pacientes que usaron placa superior con un plano anterior de mordida y tornillo de expansión seguido de un tipo de activador, donde a los 31 meses de tratamiento se corrigió las relaciones molares clase II en clase I, alineando y normalizando la mordida profunda. Poco o ningún cambio fueron detectados a nivel del ángulo ANB y de la hipodivergencia del ángulo del plano mandibularpalatal, siendo estos resultados estables después de 20 años.

Kim y Little (1999) (15), en un estudio para evaluar la estabilidad y postretención de la corrección de la mordida profunda en pacientes con maloclusión clase II - 2, hallaron que los cambios del overbite a largo plazo fueron altamente variables, siendo la posibilidad de menos del $50 \%$ el mantener un overbite menor de $4 \mathrm{~mm}$ y una tendencia a regresar a su posición inicial de mordida profunda.

Kirjavainen et al. (2007) (16), en un estudio realizado en niños con maloclusión clase II, tratados con aparato extraoral ortodóntico de tracción cervical, hallaron una disminución de la convexidad facial debido a una restricción del crecimiento maxilar en el punto A, mientras que el resto del perfil facial incluyendo la mandíbula, continuó creciendo hacia delante a un ritmo normal. Sólo con el uso del aparato de tracción extraoral cervical, se obtuvieron relaciones molares clase I en todos los casos, así como una inclinación vestibular de los incisivos su- periores.

Marchiori et al. (2007) (17), hallaron cambios en la relación molar en pacientes con diagnóstico de maloclusión clase II división 1, y tratados con aparato extraoral ortodóntico de tracción cervical (AEO), sólo o en combinación con expansión rápida maxilar (ERM), hallando un movimiento e inclinación distal de las primeras molares permanentes superiores en ambos casos, siendo mayor el movimiento en el primer caso.

\section{Conclusión}

El tratamiento temprano de la maloclusión clase II división 2 es eficiente con el uso de diferentes tipos de aparatología, siendo en este caso exitoso, el uso de una placa de expansión ortodóntica en combinación con el lip bumper y un aparato extraoral ortodóntico de tracción cervical, resultando este último útil para la distalización de las primeras molares superiores permanentes.

\section{Referencias bibliográficas}

1. King GJ, Keeling SD, Hocevar RA, Wheeler TT. The timing of treatment for Class II malocclusions in children: a literature review. Angle Orthod. 1990; 60(2):87-97.

2. Brezniak N, Arad A, Heller M, Dinbar A, Dinte A, Wasserstein A. Pathognomonic cephalometric characteristics of Angle Class II Division 2 malocclusion. Angle Orthod. 2002; 72(3):251-7.

3. Ruf S, Pancherz H. Class II Division 2 malocclusion: genetics or environment? A case report of monozygotic twins. Angle Orthod. 1999; 69(4):321-4.

4. Peck S, Peck L, Kataja M. Class II Division 2 malocclusion: a heritable pattern of small teeth in well-developed jaws. Angle Orthod. 1998; 68(1):9-20.

5. Vellini F. Ortodoncia: Diagnóstico y Planificación Clínica. 1ra Ed. Sao Paulo: Editorial Las Artes Médicas; 2002.

6. Escriván de Saturno L . Ortodoncia en Dentición Mixta. 1ra Ed. Caracas: Editorial Amolca; 2007.

7. Maj G, Lucchese FP. The mandible in class II, division 2. Angle Orthod. 1982; 52(4):28892.

8. Proffit W. Ortodoncia Contemporánea: Teoría y Práctica. 3ra Ed. Madrid: Ed. Elsevier España SA; 2001.

9. Pancherz H, Zieber K, Hoyer B. Cephalometric characteristics of Class II division 1 and Class II division 2 malocclusions: a comparative study in children. Angle Orthod. 1997; 67(2):11120.

10. Bishara S. Ortodoncia. 3ra Ed. Madrid: Ed McGraw Hill; 2003

11. Pavlow SS, McGorray SP, Taylor MG, Dolce C, King GJ, Wheeler TT. Effect of early treatment on stability of occlusion in patients with Class II malocclusion. Am J Orthod Dentofacial Orthop. 2008; 133(2):235-44.

12.Korn M, Melsen B. Early treatment with a maxillary lip bumper-bite plateau combination. Angle Orthod. 2008; 78(5):83846.

13. Murphy CC, Magness WB, English JD, Frazier-Bowers SA, Salas AM. A longitudinal study of incremental expansion using a mandibular lip bumper. Angle Orthod. 2003; 73(4):396-400.

14.Ferrazzini G. Class II/2 malocclusion: early treatment with removable appliances and stability after 20 years [Abstract]. Schweiz Monatsschr 
Zahnmed. 2008; 118(9):814-9

15. Kim TW, Little RM. Postretention assessment of deep overbite correction in Class II Division 2 malocclusion. Angle Orthod. 1999; 69(2):175-86.

16. Kirjavainen M, Hurmerinta K,
Kirjavainen T. Facial profile changes in early Class II correction with cervical headgear. Angle Orthod. 2007; 77(6):960-7.

17. Marchiori Farret M, de Lima EM, Pereira Araújo V, Deon
Rizzatto SM, Macedo de Menezes L, Lima Grossi M. Molar changes with cervical headgear alone or in combination with rapid maxillary expansion. Angle Orthod. 2008; 78(5):84751. 